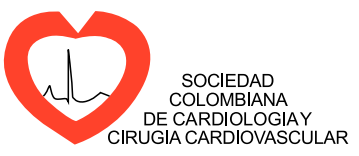

\title{
EDITORIAL
}

\section{Genomic Medicine in Cardiology}

\section{Medicina Genómica en Cardiología}

\author{
Ignacio Briceño Balcázar ${ }^{a, b}$
}

\author{
a Instituto de Genética Humana [Human Genetics Institute], Facultad de Medicina [Medicine Department], Pontificia Universidad \\ Javeriana, Bogotá, Colombia \\ b Facultad de Medicina [Medicine Department], Universidad de La Sabana, Bogotá, Colombia
}

Received 12 October 2016; accepted 17 October 2016

Available online 17 January 2017

The development of new technologies and the knowledge of the human genome sequence have opened the possibility of studying it at prices that are affordable for patients and health care institutions, allowing its application in high priority diseases such as cardiovascular diseases, diabetes and cancer.

In the last few years, large scale genome sequencing has led to the discovery of gene variants which confer risk for many common and complex diseases. A number of discoveries have been made in cardiovascular medicine of sequence variants which are associated with disorders such as coronary artery disease, atrial fibrillation, peripheral vascular disease, aortic aneurysm and ischemic stroke. Therefore, a large proportion of genetic data has been accumulated in cardiology, and this has increased the understanding of a significant number of diseases.

The study of the polymorphic genetic variants associated with a risk for different diseases creates a potential for predictive and preventive medicine. Currently, clinical laboratories offer studies of over 30,000 variants associated with genetic susceptibility, which are ever more accessible to physicians and patients. These tests allow for the development of personalized preventive health plans. While the current criteria for therapeutic management are phenotypic

DOI of original article:

http://dx.doi.org/10.1016/j.rccar.2016.10.046

E-mail address: ibriceno@javeriana.edu.co (age, body mass index, complete blood count or kidney function, for example), the individual genetic features of patients and their diseases are being neglected. Therefore, it is essential to consider the relevant genetic polymorphisms in order to improve the characterization of prevalent and incident cardiovascular diseases; both to select suitable treatment, as well as to determine the appropriate medications and doses for each patient.

There are also legal aspects in this new medical approach. For example, there is a medication legislation requiring the listing on labels of the different responses according to the patientsí genetic variants, considering that similar doses may cause adverse reactions in different individuals, while at the same time, those doses may be insufficient for some. This new regulatory scenario will promote the verification of pharmacological response polymorphisms prior to administering medications such as anticoagulants, hyperlipidemia treatments and chemotherapy, among others.

Furthermore, with regard to recessive diseases resulting from the presence of two alleles of a mutated gene inherited from both mother and father, it is possible to determine, through a study of the respective mutations, if a couple is at high risk of having children with the disease. In this way, parents may decide if they will run the risk, or carry out activities such as preimplantation diagnosis, or choose rather to adopt.

Although molecular profile genetic studies are promising, there are questions regarding the application procedure 
and how the results are communicated to the patients. Beyond their good clinical use, which, as can be seen, is highly advisable, they may be a cause for concern: a- the presymptomatic diagnosis - especially in children-, b- the intentional misuse of this information by medical insurance companies, considering genetic conditions to be preexisting conditions-, and c- the large amount of information and its relatively complex current application in populations where association studies have not yet been performed. Let us consider the implications of this $a, b$, and $c$ :

a. In several countries, this type of test is requested in pharmacies or laboratories - without a medical prescription - and the results are sent by mail directly to the interested party. It is concerning to consider what could happen to patients who receive this information without its context; for example, if the report indicates a susceptibility to Alzheimer's disease without medical support to identify the risk of its development, or the possibility of passing it on to their descendants. This suggests the importance, more than ever, of clinical and genetic counseling when delivering the results of the analysis.

b. Insurance companies should take molecular genetic profiles into account as a preventive tool to improve the quality of the service while at the same time reducing costs. In the case of anticoagulants, for example, if a prior pharmacogenetic exam is performed, the optimal medication may be applied in a timely fashion, without resorting to therapeutic trials, thus avoiding harmful side effects. c. Another aspect to keep in mind is that association studies between genetic polymorphisms and the disease require thousands of cases and controls, which makes them very costly for implementation in Colombia. Thus, the ancestral origin of the patients should be considered in the interpretation of results, in order to interpret the findings according to each one's ethnic affiliation. For now, this is the most appropriate conduct, considering that it will take a long time before validation studies may be performed in each country.

In the field of cardiology, the advent of next generation sequencing techniques has elucidated the causes of congenital structural cardiopathies and rhythm disorders at the genetic level, allowing for the examination of thousands of candidate genes associated with these diseases. This facilitates an accurate diagnosis, as well as the identification of susceptible relatives who might still be asymptomatic and who could be included in prevention programs.

In many cases, clinical genomics results offer new mechanistic clues in the pathophysiology of complex cardiovascular diseases, and may point to new therapeutic approaches. The next important step is to begin to transform these results through practical clinical knowledge in order to improve cardiovascular health care.

As with all great medical advances, genomics opens a path which will bring enormous benefits as long as the criteria of good medical practice are taken into account. 